\title{
A stochastic process model for life cycle cost analysis of nuclear power plant systems
}

\author{
J.A.M. van der Weide \\ Faculty of Electrical Engineering, Mathematics and Computer Science, Delft University of Technology, \\ Delft, The Netherlands \\ Mahesh D. Pandey \\ Department of Civil and Environmental Engineering, University of Waterloo, Waterloo, Canada
}

\begin{abstract}
The paper presents a general stochastic model to analyze the life cycle cost of an engineering system that is affected by minor but repairable failures interrupting the operation and a major failure that would require the replacement or renewal of the failed system. It is commonly observed that the frequency of minor failures increases with aging of the system due to cumulative effect of operational wear and tear. At the same time, system's vulnerability to major failures also increases with aging. The paper presents a composite stochastic process model in which the minor failures are modeled as a non-homogeneous Poisson process and the occurrences of major failure as a renewal process. The paper presents the derivation of the renewal equation for the expected cost. The age replacement policy is formulated to minimize the life cycle cost via a preventive replacement of the system.
\end{abstract}

\section{INTRODUCTION}

A nuclear plant comprises of several thousands of engineering Systems, Structures and Components (SSCs). An important aspect of nuclear asset management is to inspect, maintain and replace such a large and diverse population of SSCs to enure safe, reliable and cost-effective operation of the nuclear plant. It is realized that the nuclear plants can be safely operated over an extended period of time, beyond their initially stipulated service life of 20-30 years. The planning of Long Term Operation (LTO) by prudent planning of maintenance and replacement of critical systems is an active area of research and development in the nuclear industry.

In Canada, nuclear utilities are actively planning the refurbishment of existing stations as they approach the end of first life. Given the limited capital budget for refurbishment, how to optimize the scope of refurbishment projects is a key challenge faced by the plant managers. The reason is that uncertainty about the condition of a system, and hence its reliability in the future, confounds the decision about replacing or overhauling it at the time of refurbishment or postponing this in the far future. If it can be shown that a system can be operated with high reliability without interruptions caused by failures, then its replacement can be deferred to some future date. Otherwise, it should be prudent the system during a prolonged refurbishment outage at the station.

This decision problem is conceptualized in the following way. Suppose a system experiences minor failures which can be repaired relatively easily in a reasonable time. The most negative impact of these failures is plant outage or derating causing the losspower generation. As an example, transformers used in the power plant suffer from failures of service terminals or degradation of insulating oil, which are examples of such minor failures. The frequency of minor failures is expected to increase due to cumulative nature of aging related degradation. The system can be affected by another degradation mechanism that could cause a complete failure of the system of such a major magnitude that it cannot be repaired. The replacement of the fail system would much larger expenditure. In summary, the system experiences several minor failures with increasing frequency and the propensity of a major failure, though infrequent in nature, also increases with aging.

In this context, a dilemma for the decision maker is that if the system is not replaced at a right time, minor failures may become so frequent that total loss would become unacceptable. On the other hand, an early replacement could be inefficient use of the capital since full service life of the system is not utilized. 
The main objective of this paper is to present a stochastic process model to estimate expected life cycle cost of such a system over a finite planning horizon. The paper is organized as follows. Section 2 introduces the terminology and assumptions of the model and sets up the formulation of renewal equation. In Section 3, optimization of expected cost via an age replacement policy is presented. A practical application is illustrated in Section 4 .

\section{PROBABILISTIC ANALYSIS}

\subsection{Terminology and assumptions}

The objective is to evaluate the total expected cost of major and minor failures in a time interval $(0, t)$, also known as the planning horizon or remaining service life of the station. The schematic of the stochastic process model is shown in Figure 1.

\subsubsection{Major failures as renewal process}

The occurrence of major failures is modelled as a stochastic renewal process. The time between major failures, $Y_{1}, Y_{2}, \ldots, Y_{n}, \ldots$, are iid random variables with a cumulative distribution, $F_{Y}(y)$. After a major failure, the system is replaced by a new and identical system. Thus, a major failure marks the end of the service life as well as the time of renewal of the system. The chronological (or calendar) times of occurrence of major failures, denoted as $S_{1}, S_{2}, \ldots, S_{n}$, arepartial sums, $S_{n}=S_{n-1}+Y_{n}$, $n=1,2, \ldots$, where $S_{0}=0$.

The number of failures, $N(t)$, during the interval $(0, t)$ is an uncertain quantity, referred to as a stochastic counting process with the renewal function (Çinlar 1975, Tijms 2003):

$m_{Y}(t)=\mathbb{E}[N(t)]$.

which is a solution of the following renewal equation

$m_{Y}(t)=F_{Y}(t)+\int_{0}^{t} m_{Y}(t-y) d F_{Y}(y)$.

A suitable distribution, such as the Weibull distribution, for $Y$ can be chosen to model the increasing likelihood of failure with aging.

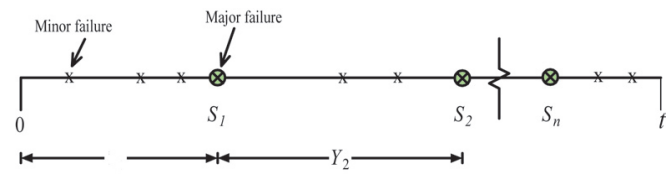

Figure 1. Stochastic process model of the system failures.

\subsubsection{Minor failures as non-homogenous poisson process}

The occurrence of minor failures during the service life, such as an interval $\left(0, S_{1}\right)$, is modelled as a Non-Homogeneous Poisson Process (NHPP), as shown in Figure 1. After a minor failure, the system is minimally repaired. It means that systemis just restored back to the working state, but the failure rate of the system remains unchanged. In other words, the aging of the system continues unabated. The NHPP model can be completely described by the mean intensity function $W(t)$, which is theexpected number of failures in $(0, t)$, i.e., $W(t)=E[K(t)], K(t)$ where is the number of failures in the interval $(0, t)$. For example, the power law process has $W(t)=a t^{b}$.

\subsection{Cost analysis}

The total cost in the interval $(0, t]$, denoted as $D(t)$, depends on the cost of major and minor failures that can take place in this interval. This interval is composed of a number of renewal cycles, and cost due to minor failures is accrued within each cycle. Consider a general $n$th cycle of duration $Y_{n}$ that starts at time $S_{n-1}$ and ends at $S_{n}$. The cost incurred in this cycle, $C_{n}$, is given as

$C_{n}=c_{m} K\left(Y_{n}\right)+c_{f}$

where $c_{m}$ and $c_{f}$ are the unit cost of minor and major failures, respectively. Since $C_{n}$ is a random variable, its expected (or average) value is derived as follows. Suppose the renewal cycle has a given duration, $Y_{n}=y$, such that the expected cost can be given as

$$
\begin{aligned}
\mathbb{E}\left[C_{n} \mid Y_{n}=y\right] & =c_{m} \mathbb{E}\left[K\left(Y_{n}\right) \mid Y_{n}=y\right]+c_{f} \\
& =c_{m} W(y)+c_{f} .
\end{aligned}
$$

By removing the conditioning, the final expression is obtained as

$$
\begin{aligned}
\mathbb{E}\left[C_{n}\right] & =\int_{0}^{\infty} \mathbb{E}\left[C_{n} \mid Y_{n}=y\right] d F_{Y}(y) \\
& =c_{f}+c_{m} \int_{0}^{\infty} W(y) d F_{Y}(y)
\end{aligned}
$$

The total cost, $D(t)$, in the interval $(0, t]$, can be written in principle as

$D(t)=\sum_{i=1}^{N(t)} C_{i}+c_{m} K\left(t-S_{N(t)}\right)$

Thus, the total cost is a sum of two parts. First, the cost incurred in $N(t)$ complete renewal cycles, as given by Eq. (4). Second, the cost of minor failures in the period between the last renewal time, $S_{N(t)}$, and the end of the interval at time $t$. 


\subsection{Integral equation for expected cost}

In this Section, a renewal-type integral equation is derived for the total expected cost in an interval $(0, t]$, denoted as $\mathbb{E}[D(t)]=\bar{D}(t)$. The derivation begins with the supposition that the first renewal takes place at time $Y_{1}=y$, which can be either within the planning horizon $t$ or beyond. Thus, using these two disjoint events, $Y_{1} \leq t$ or $Y_{1}>t$, along with the law of total expectation, the expected cost can be written as

$$
\bar{D}(t)=\mathbb{E}\left[D(t) ; Y_{1} \leq t\right]+\mathbb{E}\left[D(t) ; Y_{1}>t\right]
$$

The subsequent analysis is essentially the simplification of the two joint expectations.

Let's begin with the second expectation term which involves only the cost of minor failures. Thus,

$$
\begin{aligned}
\mathbb{E}\left[D(t) ; Y_{1}>t\right] & =\mathbb{E}\left[c_{m} K(t) ; Y_{1}>t\right] \\
& =c_{m} W(t)\left(1-F_{Y}(t)\right) .
\end{aligned}
$$

To analyze the first expectation term, the law of total expectation is used as follows:

$\mathbb{E}\left[D(t) ; Y_{1} \leq t\right]=\int_{0}^{t} \mathbb{E}\left[D(t) \mid Y_{1}=y\right] d F_{Y}(y)$.

Given $Y_{1}=y$, the total cost is a sum of the cost in first renewal cycle, $C_{1}$, and that in the remaining time span, $(y, t]$. Therefore,

$$
\mathbb{E}\left[D(t) \mid Y_{1}=y\right]=\mathbb{E}\left[C_{1}+D(y, t) \mid Y_{1}=y\right],
$$

where $D(y, t)$ denotes the cost over the interval $(y, t]$. This can be simplified using the linearity of expectation:

$$
\begin{aligned}
\mathbb{E}\left[C_{1}+D(y, t) \mid Y_{1}=y\right] \\
=\mathbb{E}\left[C_{1} \mid Y_{1}=y\right]+\mathbb{E}\left[D(y, t) \mid Y_{1}=y\right]
\end{aligned}
$$

After a major failure at $Y_{1}=y$, the renewal by an identical component takes place. This implies that the renewal process, independent of the history, restarts again with the time origin $y$. The following equality in distribution can be concluded: $D(y, t) \stackrel{d}{=} B(t-y)$. This is known as the "renewal argument" in the theory of stochastic renewal processes (Tijms 2003), which allows to simplify the the second expectation as

$$
\begin{aligned}
\mathbb{E}\left[D(y, t) \mid Y_{1}=y\right] & =\mathbb{E}\left[D(t-y) \mid Y_{1}=y\right] \\
& =\bar{D}(t-y) .
\end{aligned}
$$

Recall that $C_{1}$ is given by Eq. (4) and substituting from Eq. (11) in Eq. (8), leads to

$$
\begin{aligned}
\mathbb{E}\left[D(t) ; Y_{1} \leq t\right]= & \int_{0}^{t}\left(c_{m} W(y)+c_{f}\right) d F_{Y}(y) \\
& +\int_{0}^{t} \bar{D}(t-y) d F_{Y}(y)
\end{aligned}
$$

Now substituting into Eq. (6) from (12) and (7), the following integral equation for expected cost is obtained.

$\mathbb{E}[D(t)]=\int_{0}^{t} \bar{D}(t-y) d F_{Y}(y)+G(t)$

where

$$
\begin{aligned}
G(t)= & \int_{0}^{t}\left(c_{m} W(y)+c_{f}\right) d F_{y}(y) \\
& +c_{m} W(t)\left(1-F_{Y}(t)\right)
\end{aligned}
$$

\section{OPTIMIZATION OF EXPECTED COST}

\subsection{Age replacement policy}

A sudden failure, major or minor, interrupts the plant operation and burdens the staff with managing unplanned outages. To minimize adverse impact of sudden failures, preventive replacement policies can be adopted. This Section presents the age replacement policy, in which a system is preventively replaced at a cost $c_{p}$ as soon as it reaches an age of $t_{r}$. Of course this policy does not guarantee against a major failure before the age $t_{r}$. The system is also renewed at a $\operatorname{cost} c_{f}$, should a major failure suddenly occurs before the age $t_{r}$. Since the cost of preventive replacement tends to be much lower than that of sudden failure $\left(c_{p}<c_{f}\right)$, it is possible to find an optimal age of replacement that would minimize the total cost in a time interval $(0, t]$.

\subsection{Definition of the renewal cycle}

The renewal cycle duration under the age replacement policy is given as, $X=\min \left(Y, t_{r}\right)$, and its distribution function can be defined as

$F_{X}(x)=F_{Y}(x) \mathbf{1}_{\left[0, t_{r}\right)}(x)+\mathbf{1}_{\left[t_{r}, \infty\right)}(x)$,

where the indicator function of a set $A$ is defined as $\mathbf{1}_{A}(x)=1$ if $x \in A$ and $\mathbf{1}_{A}(x)=0$ if $x \notin A$. The expected cycle length is given as

$\mathbb{E}[X]=\int_{0}^{t_{r}} y d F_{Y}(y)+t_{r}\left(1-F_{Y}\left(t_{r}\right)\right)$. 
The total cost in a renewal cycle of duration $X$ is a sum of costs of minor failures and the cost of either a major failure or the cost of a preventive replacement, which can be written as

$C_{n}=K(X) c_{m}+c_{f} \mathbf{1}_{\left\{Y \leq t_{r}\right\}}+c_{p} \mathbf{1}_{\left\{Y>t_{r}\right\}}$.

Similar to Eq. (5), expected cycle cost can be given as

$$
\begin{aligned}
\mathbb{E}\left[C_{n}\right]= & c_{m} \int_{0}^{\infty} W(x) d F_{X}(x) \\
& +c_{f} F_{Y}\left(t_{r}\right)+c_{p}\left(1-F_{Y}\left(t_{r}\right)\right)
\end{aligned}
$$

Because of the nature of age replacement policy, the integration can be simplified as

$$
\begin{aligned}
\int_{0}^{\infty} W(x) d F_{X}(x)= & \int_{0}^{t_{r}} W(x) d F_{Y}(x) \\
& +W\left(t_{r}\right)\left(1-F_{Y}\left(t_{r}\right)\right),
\end{aligned}
$$

\subsection{Integral equation}

The derivation of the expected cost follows the same steps as those described in Section 2.3, except that the renewal cycle distribution $F_{X}(x)$ needs to be aptly incorporated in the analysis. The expected cost $\bar{D}(t)$, conditioned on, $X_{1}$, the time of first renewal, is written as sum of two expectations, as shown in Eq. (6). It should be noted that the renewal cycle length can never exceed $t_{r}$.

In case of $X_{1}>t$ and $t<t_{r}$, the cost is given as $D(t)=K(t) c_{m}$, and its expectation is given as

$$
\begin{aligned}
\mathbb{E}\left[D(t) ; X_{1}>t\right] & =\mathbb{E}\left[K(t) c_{m} ; X_{1}>t\right] \\
& =c_{m} \mathbb{E}[K(t)] \mathbb{P}\left(X_{1}>t\right) \\
& =c_{m} W(t)\left(1-F_{X}(t)\right) .
\end{aligned}
$$

This formula is also correct for $t \geq t_{r}$, since $F_{X}(t)=1$.

Consider the analysis of the second term as

$$
\begin{aligned}
\mathbb{E}\left[D(t) ; X_{1} \leq t\right] & \left.=\mathbb{E}\left[\mathbb{E}\left(D(t) \mid X_{1}\right) \mathbf{1}_{\left\{X_{1} \leq t\right\}}\right)\right] \\
& =\int_{0}^{t} \mathbb{E}\left(D(t) \mid X_{1}=x\right) d F_{X}(x),
\end{aligned}
$$

Based on the renewal argument

$$
\begin{aligned}
& \mathbb{E}\left[\left(D(t) \mid X_{1}=x\right)\right] \\
& \quad=\mathbb{E}\left[\left(C_{1} \mid X_{1}=x\right)\right]+\bar{D}(t-x),
\end{aligned}
$$

The expected cost in the first renewal cycle can be given as

$$
\mathbb{E}\left(C_{1} \mid X_{1}=x\right)= \begin{cases}c_{m} W(x)+c_{f}, & x<t_{r}, \\ c_{m} W\left(t_{r}\right)+c_{p} & x=t_{r} .\end{cases}
$$

Substituting from Eq. (21) and (22) into (20) leads to

$$
\begin{aligned}
\mathbb{E}\left[D(t) ; X_{1} \leq t\right]= & \int_{0}^{t}\left[W(x) c_{m}+\left(c_{f}-c_{p}\right) 1_{\left[0, t_{r}\right)}(x)\right. \\
& \left.+c_{p} 1_{\left[0, t_{r}\right]}(x)\right] d F_{X}(x) \\
& +\int_{0}^{t} \bar{D}(t-x) d F_{X}(x)
\end{aligned}
$$

Similar to Eq. (6), Eq. (19) and (22), the final integral equation for expected cost can be written as

$$
\bar{D}(t)=G(t)+\int_{0}^{t} \bar{D}(t-x) d F_{X}(x),
$$

where

$$
\begin{aligned}
G(t)= & c_{m} W(t)\left(1-F_{X}(t)\right)+\int_{0}^{t}\left[W(x) c_{m}\right. \\
& \left.+\left(c_{f}-c_{p}\right) 1_{\left[0, t_{r}\right)}(x)+c_{p} 1_{\left[0, t_{r}\right]}(x)\right] d F_{X}(x) .
\end{aligned}
$$

The renewal equation (24) has a unique, locally bounded solution, since $G(t)$ is locally bounded. This solution is given as

$\bar{D}(t)=G(t)+\int_{0}^{t} G(t-x) d m_{X}(x)$,

where $m_{X}$ is the renewal function for a renewal process with inter-occurrence time $X$ as defined by (1).

\section{APPLICATION}

\subsection{Input data}

A sizable fleet of air compressors are installed in a nuclear plant to supply instrument air to a variety of systems important to safety. This example focuses on quantifying the life cycle cost of compressors that are vulnerable to minor and major failures. Based on the operational history of failures and maintenance expenditures, the following input data and models were estimated.

The time to major failure of a compressor is modeled as the Weibull distribution with distribution function, $F(x)=1-\exp \left(-(x / \beta)^{\alpha}\right)$. The distribution parameters we estimated as $\alpha=2.5$ and $\beta=25$ year, which correspond to the mean life time of 22 years and coefficient of variation of 0.42 . The NHPP model for minor failures has the mean intensity function, $W(t)=a t^{b}$, with parameters estimated as $a=0.05$ and $b=2$.

The cost of a minor failure is $\$ 10,000$ and that of a major failure is $\$ 140,000$. The cost of preventive replacement is estimated as $\$ 80,000$. 


\subsection{Numerical results}

The expected cumulative cost over 30 year service life is plotted in Figure 2. It shows that in 30 year life time, expected cost is 442 thousand ( $\mathrm{K} \$$ ), as shown by the last point in Figure 2 .

The results of age replacement policy are presented in Figure 3, where the replacement age is plotted against the total expected cost over a 30 -year period. It is interesting to see that the life cycle cost can be significantly minimized by choosing an appropriate replacement age. For example, if a compressor were replaced at the age of 11 year, the life cycle cost would be reduced to $343 \mathrm{~K} \$$, which amounts to a reduction of $22 \%$ in the total cost as compared to the base case in which the compressor is replaced upon a major failure only (i.e., no preventive replacement).

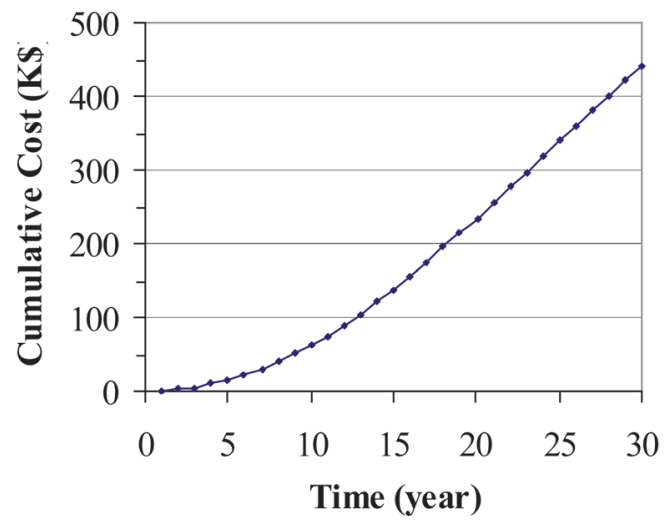

Figure 2. Cumulative expected cost over a 30 year period.

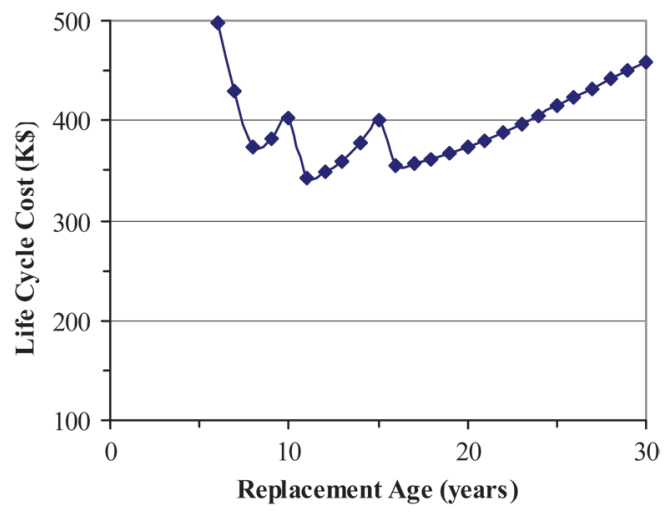

Figure 3. Replacement age versus the expected life cycle cost over a 30 year period.

\section{CONCLUSIONS}

In this paper, a probabilistic model is presented to estimate expected life cycle cost of an engineering system affected by random occurrences of both minor and major failures. A new feature of the proposed model is that it combines a nonhomogeneous Poisson process of minor failures with a renewal process of major failures. A renewal type integral equation is derived for the expected cost. In addition, a formulation of age replacement policy is presented, which has not yet been reported in the literature.

A practical example of air compressor life cycle cost analysis is presented, which shows that the life cycle cost can be significantly optimized by choosing a right age of preventive replacement. The proposed model is generic in nature and it can be applied for the asset management of nuclear plant systems, structures and components.

\section{REFERENCES}

[1] E. Çinlar, Introduction to Stochastic Processes, Prentice-Hall (1975)

[2] H.C. Tijms, A First Course in Stochastic Models, John Wiley \& Sons Ltd., West Sussex, England (2003). 